\title{
The Metamorphosis of Radionuclide Production and Development at Paul Scherrer Institute
}

\author{
P. V. Grundler ${ }^{a}$, R. Eichler ${ }^{b}$, Z. Talipa ${ }^{a}$ P. A. Schubiger ${ }^{a c}$, R. Schibliac , and N. P. van der Meulen ${ }^{\star a b}$
}

\begin{abstract}
Radionuclide production and development has a long history at the Paul Scherrer Institute (PSI) and dates back to the founding times of its forerunner institutions: the Federal Institute for Reactor Research and the Swiss Institute for Nuclear Research. The facilities used for this purpose have evolved substantially over the last five decades. Many radiometals in use today, as radiopharmaceuticals, are for the diagnosis and treatment of disease, with the most popular means of detection being Positron Emission Tomography. These positron emitters are easily produced at low proton energies using medical cyclotrons, however, developments at these facilities are lacking. Currently, the fixed $72 \mathrm{MeV}$ proton beam at PSI is degraded at IP2 irradiation station to provide the desired energy to irradiate targets to produce the likes of ${ }^{44} \mathrm{Sc},{ }^{43} \mathrm{Sc}$ and ${ }^{64} \mathrm{Cu}$ as a proof of principle, which are of great interest to the nuclear medicine community. This development work can then be implemented at facilities containing medical cyclotrons. A history of the development of radionuclides at PSI, along with current development and projects with partner institutions, is described.
\end{abstract}

Keywords: History of science $\cdot$ Radiochemistry $\cdot$ Radionuclide development $\cdot$ Radionuclide production . Radiopharmacy

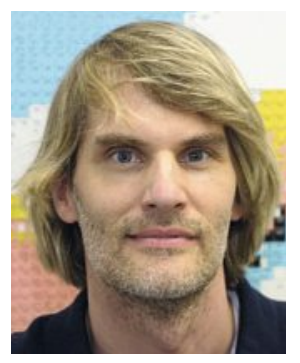

Pascal V. Grundler studied chemistry at the University of Lausanne and graduated with a diploma thesis on the catalytic properties of ruthenium aqua ions. He then moved on to investigate the reaction mechanisms of aquacarbonyl complexes of Mn, Tc and Re under the supervision of Prof. A. E. Merbach earning him a PhD, from the EPFL in 2005. In 2006, he joined the staff of the Minerals, Metals \& Solutions group at the University of Adelaide and the South Australian Museum, Australia. There, he worked on the speciation and solubility of metals in hydrothermal systems relevant to geological processes. In 2011, he returned to Switzerland to work at the Laboratory for Nuclear Materials, PSI, on chemical aspects of stress corrosion cracking (SCC) mitigation in boiling water reactors using noble metals and hydrogen. In 2019, he joined the Radionuclide Development group at PSI to work on developing targetry and separation processes.

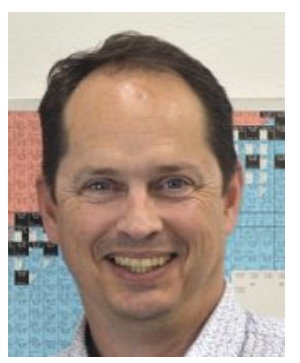

Nicholas P. van der Meulen studied chemistry at Stellenbosch University, South Africa, obtaining his $\mathrm{PhD}$ in the development of radionuclides from medium-energy proton beams in 2008. He worked at iThemba LABS, South Africa, as a research chemist in the Radionuclide Production group for 17 years before accepting the position of Group Leader of Radionuclide Development at PSI in 2013.

\section{Introduction}

The use of radionuclides has proven to be essential in nuclear medicine for the diagnosis and treatment of disease, predominantly cancer. Radiopharmaceutical research aims at developing new diagnostic and therapeutic compounds and, for this, it relies on a vector able to introduce potentially interesting radionuclides (due to their decay properties) into the tumor. Various radionuclides used in diagnosis/imaging of disease (often utilizing Positron Emission Tomography, or PET) are coming to the fore as a result of chelator development. Radionuclides can be handpicked as a result of which chelator is desired, taking the half-life and decay character of the nuclide into consideration. ${ }^{68} \mathrm{Ga}\left(\mathrm{T}_{1 / 2}=68 \mathrm{~min}\right)$, for instance, can be used for chelators that have a short biological half-life (e.g. FAPI), while ${ }^{44 / 43} \mathrm{Sc}\left(\mathrm{T}_{1 / 2}=\right.$ $\sim 4 \mathrm{~h}$ ) can be used for late-time-point imaging, also using albumin-binding chelators. The same can be said for ${ }^{61} \mathrm{Cu}\left(\mathrm{T}_{1 / 2}=3 \mathrm{~h}\right)$ and ${ }^{64} \mathrm{Cu}\left(\mathrm{T}_{1 / 2}=12.7 \mathrm{~h}\right) .{ }^{155} \mathrm{~Tb}$, which can be used for imaging of disease using Single Photon Emission Computed Tomography (SPECT), can be used as a theranostic match to ${ }^{161} \mathrm{~Tb}$ (a potentially effective therapeutic nuclide), therefore, what you image is what you treat (same element, therefore, same chemical properties). In this case, the aim is to use the same biological vector for diagnostic and therapy by labelling it with one of two radionuclides of the same element (or two elements with very similar chemical behavior, e.g. lanthanides) with adequate decay properties. The Radionuclide Development group at PSI aims to explore the production of such radionuclides and then provide them in a quantity and quality suitable for preclinical research and potential clinical studies.

\footnotetext{
${ }^{*}$ Correspondence: Dr. N. P. van der Meulen ${ }^{\text {ab }}$, E-mail: nick.vandermeulen@psi.ch 
The two main routes for nuclide production are irradiation either with charged particles (protons, deuterons and $\alpha$-particles) or with neutrons. Depending on the particle beam energy and the capture cross section of the target nuclei, different nuclear reactions can take place and produce nuclides in different yields. In addition, it is sometimes necessary to use enriched target material to obtain a product of high quality and quantity. As many of these enriched materials are expensive, it is necessary to devise a means of recycling the target material for re-use. Chemical separation after irradiation is generally required, since only a small fraction of the target is converted in the nuclear reaction and this requires isolation to ensure a chemically pure product.

Radionuclides obtained by proton irradiation are neutron poor and tend to decay by positron $\left(\beta^{+}\right)$emission or by electron capture $(\in)$, usually followed by gamma $(\gamma)$ emission. They are, thus, particularly suitable for the diagnosis of disease, where the most popular means of detection is Positron Emission Tomography (PET). These positron emitters can be produced at medical cyclotrons (with an installed solid target station) utilizing the (p,n) nuclear reaction in the range of $10-25 \mathrm{MeV}$ protons. Development work using such facilities is rare, however.

Paul Scherrer Institute (PSI) has made a substantial contribution to the world of nuclear medicine over the years by producing radionuclides, as well as radiopharmaceuticals. PSI was formed from the two initial facilities Federal Reactor Research Institute (EIR, established in the 1950s) and Swiss Institute for Nuclear Research (SIN, established in the late 1960s) and continued their radionuclide/radiopharmaceutical production programme. The facilities used towards this programme have evolved over the last five decades and its history is described briefly here. This historical development has established radionuclide development as a major education and research driver towards radiochemistry in Switzerland.

\subsection{Radionuclide Production for Medical Use}

The development and production of novel radiometals for radiopharmaceutical use is a multidisciplinary affair, requiring input from nuclear physicists, material scientists, radiochemists, engineers, radiopharmacists, immunologists, structural biologists, medical physicists, and medical doctors to ensure success. As the radionuclide forms only the initial step of this radiopharmaceutical/medical chain, it is vital to have collaboration between all these disciplines for the radionuclide in question to reach the clinic. ${ }^{[1]}$ The production of radionuclides towards medical applications has four important multidisciplinary constituents, namely, i) particle accelerator or reactor operation (physics, engineering), ii) irradiation physics and chemistry (including production cross-section, particle matter interaction, target preparation, material sciences and engineering), iii) chemical separation (radiochemistry, physical chemistry, radioanalytics) and iv) quality control (bio-inorganic chemistry, dosimetry) of the final product. If new radionuclides are developed, even their fundamental decay data have to be assessed quantitatively. The choice of radionuclide for the chelator in question will depend on radiation characteristics such as the type of decay emission, the energy of the emission(s), the linear energy transfer of the particles (for therapeutic nuclides) and physical half-life. If a gamma-ray photon is emitted in the decay process, its energy and branching ratio should be in the diagnostically useful range $(<$ $400 \mathrm{keV}$ ). Should a positron-emitting radionuclide be chosen, it is important to keep gamma emission, other than resulting from annihilation, to a minimum, to minimize dose to the patient. The physical half-life of therapeutic radionuclides is extremely important for minimizing decay loss. Here, we focus on the chemical aspects of this complex research scheme pursuing the production and development of radiometals for radiopharmaceutical application.

\subsubsection{Choice and Preparation of Target Material}

Targetry is an important step towards radionuclide production and development. The choice of target material has a vital role to play as it will determine the irradiation parameters, as well as the subsequent devised chemical separation. Radiometals are predominantly produced using solid target systems. Solid targets are prepared by sintering, rolling, melting, sedimenting, mechanical pressing and electrodeposition, among others. ${ }^{[1]}$

Should the element be chemically stable under environmental conditions, it can be used as target material in pure form. Should the element in question not be stable, however, the oxide thereof has proven to be a good alternative. The use of other salts can be problematic, either due to activation of the elements in the counter-ion or decomposition with formation of gaseous products (such as via the ${ }^{16} \mathrm{O}(\mathrm{p}, \alpha){ }^{13} \mathrm{~N}$ nuclear reaction) in the case of carbonates, nitrates or hydrated salts. ${ }^{[2]}$

Lower proton energies are used to irradiate and produce PET radiometals, thereby, justifying the use of medical cyclotrons. Radionuclidic purity of products for nuclear medicine purposes is fundamental and one, sometimes, has to turn to the use of enriched target material to prevent undesired nuclear side-reactions, which can negatively affect the quality of the desired product. As much as the nuclidic purity of the starting material is critical for the quality of the final product, an accurate knowledge of the cross sections for neutron or proton capture is essential to minimize the production of unwanted nuclides due to nuclear side reactions. Enriched material is generally expensive and one has to be able to optimize the targets such that enough activity is produced from as little material as possible and perform recycling of the target material post production.

\subsubsection{Post-irradiation Processing}

Only a small proportion of the target material undergoes the nuclear reaction during irradiation. As a result, the desired radionuclide needs to be separated from the target material in question. Furthermore, the final product also has to be free of contaminants that can compete with the radiometal for the labelling of the biologically active molecules used in nuclear medicine applications. Thus, in addition to finding the optimal irradiation conditions, radionuclide development also implies establishing a fast and highly efficient post-irradiation chemical separation and purification procedure. To achieve this goal under high radiation dose conditions, the use of ion exchange and extraction resins has proven to be effective. Typically, after irradiation and removal from the irradiation capsule (or quartz ampoule in the case of neutron irradiation) the target is dissolved with an appropriate acid solution. This solution may be further processed to make it compatible with the optimum conditions necessary for effective separation on the chosen ion exchange resin. Finally, to achieve high activity per volume product, the fraction collected from the ion exchanger containing the desired purified radionuclide is loaded onto an extraction resin, from which it can be eluted in a small volume. To perform all the procedures described, specific chemical separation panels have been developed at PSI such that they can be operated in hot cells with manipulators (Fig. 1).

\section{Radionuclide Production at the PSI Site: The Early Years}

The Swiss Atomic Energy Study Commission (SKA) was created soon after World War II to follow the developments related to nuclear energy and was headed by Prof. Paul Scherrer from ETH Zurich (ETHZ). Considerations regarding the sourcing of the required materials for a reactor led to the idea of developing a reactor running on natural uranium and moderated with heavy water. Unlike in other countries, industrial companies were soon taking the lead and in 1955, with Scherrer as the driving force, Reaktor AG - a private company - was founded and established 


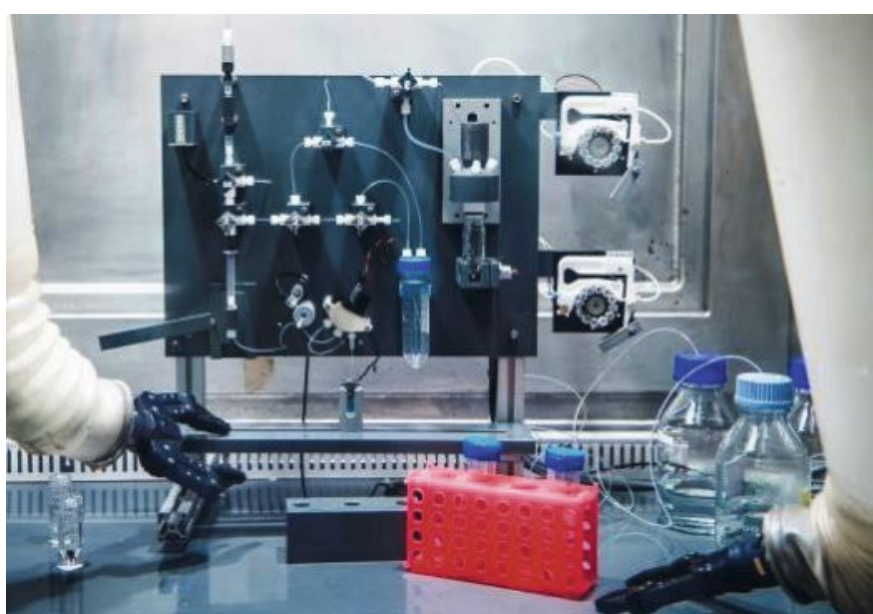

Fig. 1. Representative chemical separation panel used in a hot cell to produce radionuclides at PSI.

in Würenlingen on the site where the PSI East is located today. Its purpose was to develop the nuclear industry in Switzerland. [3]

Also in 1955, the United States Atomic Energy Commission set up a reactor at the International Conference on the Peaceful Uses of Atomic Energy, in Geneva. This 'aquarium reactor' was run at the conference site with enriched uranium as a proof of principle. Paul Scherrer and his industrial partners realized the bargain they could make, since sending the reactor back to the US, now that it had been critical, would be impractical. They purchased it for less than the material costs and transferred it to the site of Reaktor AG. ${ }^{[4]}$ It was renamed SAPHIR and went critical again in Würenlingen in April 1957. This reactor was able to handle and produce small quantities of radionuclides for the medical and research fraternities. ${ }^{[5]}$

At the beginning of 1960, the ownership of the facilities in Würenlingen was transferred to the Swiss Confederation and the EIR was founded. Soon after the transfer, the newly developed heavy water reactor (referred to as DIORIT) went critical in August 1960. This unit was able to manufacture large activities of radionuclides for commercial purposes. Both reactors were involved in the production of ${ }^{131} \mathrm{I}$ and radiopharmaceuticals thereof were manufactured on site and sold commercially.

\subsection{Injector I and the IP1 Irradiation Station}

In 1968, the Swiss Institute for Nuclear Research (SIN) was founded in Villigen, adjacent to EIR, just across the Aare River. The High Intensity Proton Accelerator facility was built for acceleratorbased nuclear physics research, along with radionuclide production. SIN began their radionuclide production programme in 1974/75, [6] which resulted in a collaboration with EIR for many years before the two institutions were united and renamed Paul Scherrer Institute in 1988. Injector I cyclotron was instrumental in the programme from 1974 to 1985 . This Phillips cyclotron produced $72 \mathrm{MeV}$ protons (as well as $<65 \mathrm{MeV}$ deuterons, $<130 \mathrm{MeV}$ alpha particles) ${ }^{[6,7]}$ and was initially used to boost the Ring cyclotron from 1974 to 1985, when the Cockroft-Walton and Injector II accelerators came into service and took over this role (Fig. 2). Over this period and beyond, Injector I was also utilized for commercial radionuclide production (vast activities of ${ }^{123} \mathrm{I}$, along with ${ }^{82} \mathrm{Sr}$ ), as well as for research on heavy elements and nuclear physics. This continued until 2010, after which it was retired and subsequently dismantled.

Some production, along with research and development, highlights are briefly outlined in this manuscript.

\subsection{1 ${ }^{123}$}

This radionuclide was regularly used in clinics from the 1970s to the 1990 s. It is a $\gamma$-emitter $(E=159 \mathrm{keV})$ and decays $\left(\mathrm{T}_{1 / 2}=13.2\right.$ h) via electron capture. The emitted $159 \mathrm{keV}$ photons are well

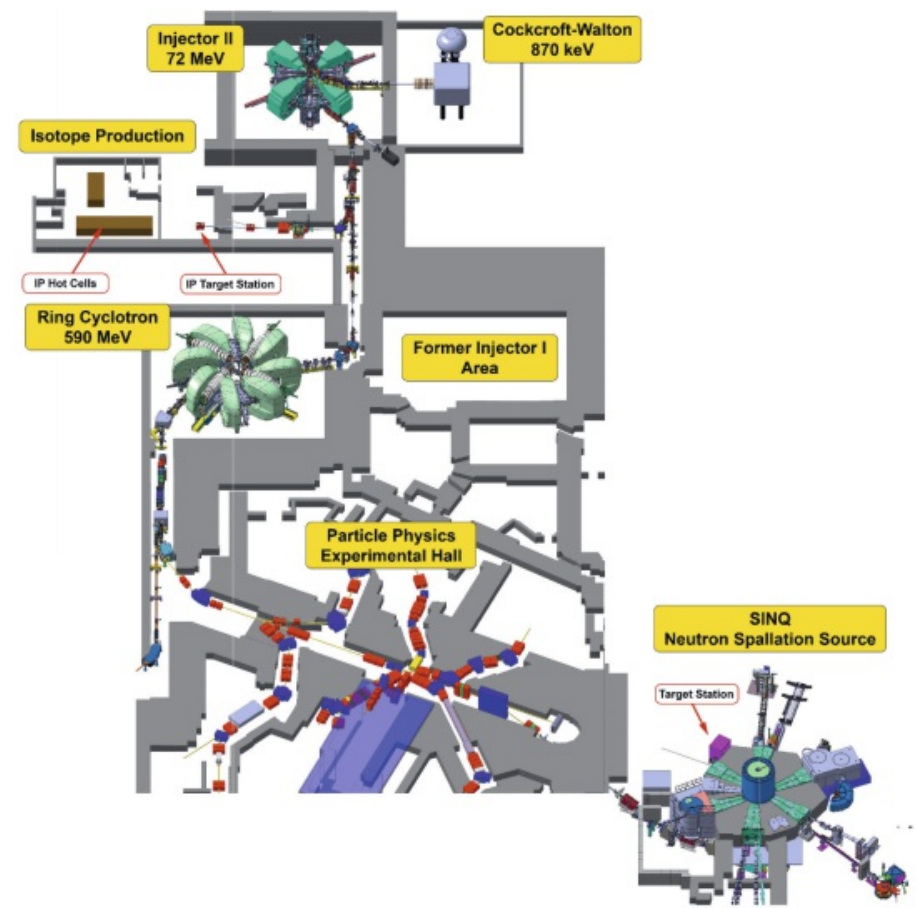

Fig. 2. High Intensity Proton Accelerator (HIPA) facility at PSI. A portion of the beam leaving Injector II is split, diverted to provide protons for the IP2 target station. Other targets can be irradiated with neutrons at a station at SINQ.

suited for imaging (scintigraphy, SPECT). PSI's Injector I (from 1974) and Injector II (from 1986) cyclotrons $^{[8]}$ were utilized for the necessary ${ }^{127} \mathrm{I}(\mathrm{p}, 5 \mathrm{n}){ }^{123} \mathrm{Xe} \rightarrow{ }^{123} \mathrm{I}$ nuclear reaction to produce the desired product. The ${ }^{\text {nat }}$ I required for the nuclear reaction was prepared as a NaI target (Fig. 3). The first batch was produced at the end of 1974 at SIN. ${ }^{[9]}$ A year later, the EIR-SIN partnership was able to produce the best quality ${ }^{123}$ I available in Europe at that time on a weekly basis. ${ }^{[10]}$

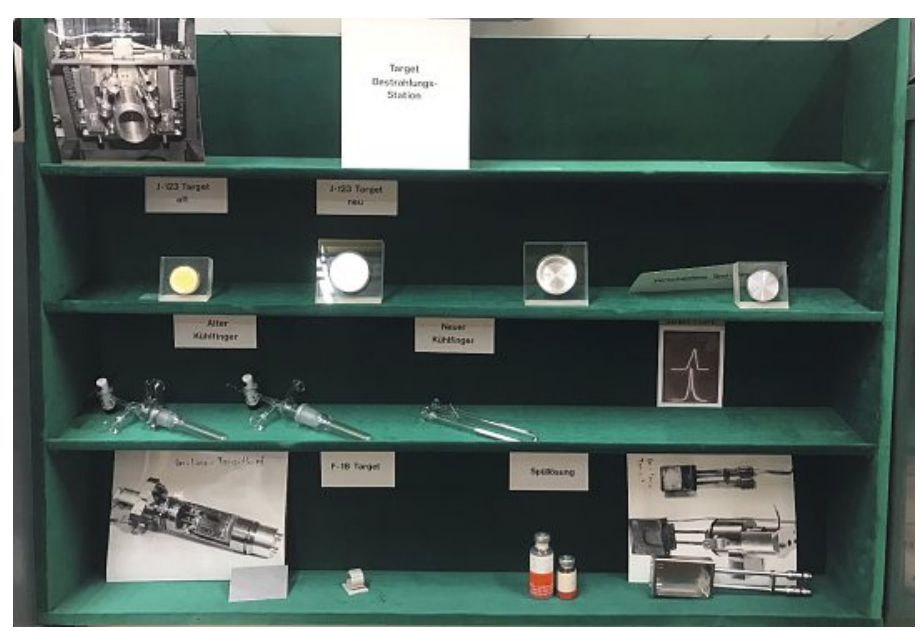

Fig. 3. Evolution of Nal targets for ${ }^{123}$ production, as well as targets designed for ${ }^{18} \mathrm{~F}$ irradiation. The 'cold finger' ('Kühlfinger') process was a means of trapping the ${ }^{123} \mathrm{Xe}$ such that it decayed to ${ }^{123}$.

There were two variations of the production developed, referred to as the 'online' and 'offline' processes. The offline process consisted of the NaI target being irradiated and then dissolved in $\mathrm{HCl}$, with the released Xe gas collected in the 'cold finger' (Fig. 4). An online method was developed, with the use of Injector I and its IP1 irradiation station, where irradiation of the rectangular target box resulted in molten $\mathrm{NaI}$ target material (after initial electrical 
heating, the power dissipation from the proton beam maintained the target in the molten state). Thermal convection in the target was achieved, as a result of painstaking beam positioning, which ensured the release of Xe from the melt. The ${ }^{123} \mathrm{Xe}$ gas released was swept away with a He stream through a 40 m pipe to a cold trap system, where it was retained and left to decay to the desired product. ${ }^{[6]}$

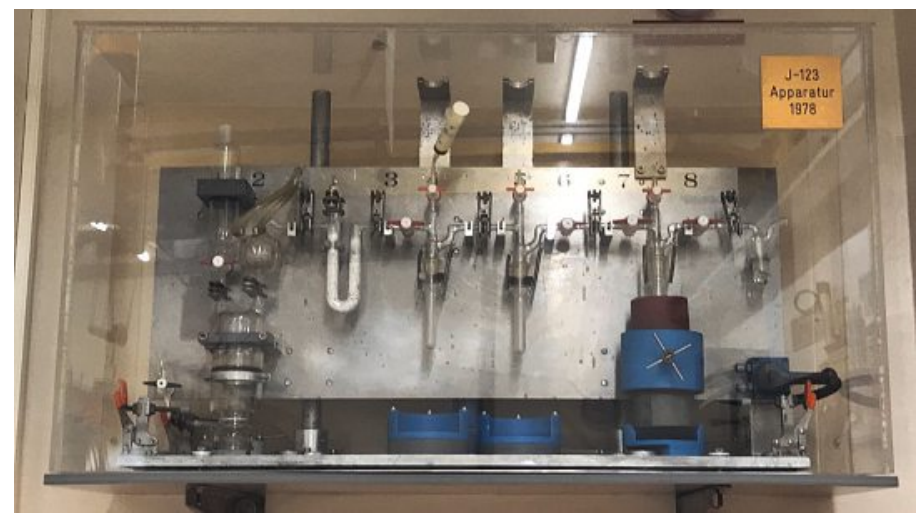

Fig. 4. ${ }^{123}$ chemical separation system, developed in 1978.

\subsection{2 ${ }^{81} R b$}

This radionuclide $\left(\mathrm{T}_{1 / 2}=4.6 \mathrm{~h}\right)$ has been used for lung perfusion studies, although it has fallen out of favor and is seldom used today. A $\sim 7 \mathrm{~g}$ natural $\mathrm{RbCl}$ pellet was encapsulated in $\mathrm{Al}$ and irradiated at $\sim 67 \mathrm{MeV}$. Two simultaneous nuclear reactions are taking place under these conditions: ${ }^{85} \mathrm{Rb}(\mathrm{p}, 5 \mathrm{n}){ }^{81} \mathrm{Sr} \rightarrow{ }^{81} \mathrm{Rb}$ and ${ }^{85} \mathrm{Rb}(\mathrm{p}, \mathrm{p}, 4 \mathrm{n}){ }^{81} \mathrm{Rb}$. Once removed from the capsule, the target was dissolved in water and the appropriate volume loaded onto a generator containing Dowex 50W-X8 cation exchange resin. ${ }^{6}$ The generator was air dried in preparation for use in clinical applications.

\subsection{3 ${ }^{82} \mathrm{Sr}$}

The production of ${ }^{82} \mathrm{Sr}\left(\mathrm{T}_{1 / 2}=25.5 \mathrm{~d}\right)$ utilized a similar concept to that of ${ }^{81} \mathrm{Rb}$ manufacture. The $\mathrm{Rb}$ salt target was irradiated with no degradation of the $72 \mathrm{MeV}$ proton beam. ${ }^{[8]}$ The concept was also performed at the IP2 target station and tests using Rb metal as target material later attempted. ${ }^{[11]} \mathrm{Rb}$ targets were irradiated and $\mathrm{Sr}$ recovered by loading an ammonia buffer solution, $\mathrm{pH} 8-9$, through a column containing Chelex 100 chelating resin. ${ }^{[12]}$ After rinsing the column with more buffer, the product was finally eluted with $0.1 \mathrm{~N} \mathrm{HCl}$. The use of $\mathrm{Rb}$ metal required dissolution in n-butanol, with the addition of ethanol, before addition of $5 \mathrm{~N} \mathrm{HCl}$ and evaporation to dryness. ${ }^{[11]}$ The activity was picked up in ammonia buffer solution and the separation performed as above. Routine production of the metal target irradiation route began in early 1993 . A generator for ${ }^{82} \mathrm{Sr} /{ }^{82} \mathrm{Rb}$ was developed ${ }^{[12]}$ towards heart infarction studies using PET. This diagnostic application is still popular in the United States today.

\subsection{4 ${ }^{18} \mathrm{~F}$}

Much development work was performed at PSI towards the production of ${ }^{18} \mathrm{~F},{ }^{[13]}$ seen today as the gold standard for PET imaging. The radionuclide is, arguably, the most common available today, as it is easily produced at medical cyclotrons utilizing the ${ }^{18} \mathrm{O}(\mathrm{p}, \mathrm{n}){ }^{18} \mathrm{~F}$ nuclear reaction. This production route was developed using a water target constructed of silver, with an internal volume of $2.6 \mathrm{~mL}$ (Fig. 3). Another means of producing the nuclide was investigated with the irradiation of a $\mathrm{Ne}$ gas target using the ${ }^{20} \mathrm{Ne}(\mathrm{p}, \mathrm{x}){ }^{18} \mathrm{~F}$ nuclear reaction, however, a two-shot protocol was observed, where the initial proton bombardment of the $\mathrm{Ne}$ gas target was followed by the irradiation of the F/Ne mixture.

\section{PSI: From 1988 to Present}

Today, PSI operates, as part of its Large Facilities, a High Intensity Proton Accelerator (HIPA) facility where three accelerators are connected in series to increase proton beam energy. Initially, a Cockroft-Walton accelerator accelerates protons at $870 \mathrm{keV}$, which are then fed into the Injector II separated-sector cyclotron. There, the protons are accelerated to $72 \mathrm{MeV}$, at a beam intensity of $\sim 2.5 \mathrm{~mA}$, en route to a larger cyclotron referred to as the 'Ring' cyclotron. Finally, the Ring cyclotron accelerates the protons further to $590 \mathrm{MeV}$ and they are then sent down the beam line to various experimental vaults. The remainder of the beam is collected in a $\mathrm{Pb}$ beam dump, which serves as a neutron spallation source for the Swiss Neutron Source (SINQ). ${ }^{[14]}$ This chain provides protons and neutrons, which can be used for producing radionuclides (Fig. 2).

\subsection{Injector II and the IP2 Irradiation Station}

The IP2 irradiation station was designed and built in the early 1980 s and was put into service in $1986 .{ }^{[7]}$ The station was designed to handle $100 \mu \mathrm{A}$ proton beam intensity and would receive beam, parasitically, from Injector II (Fig. 2) which was brought into service in 1985 and took over the leading role in the radionuclide production, and subsequent development, programme. ${ }^{[8]}$ The target holders used initially at IP2 were large, heavy and constructed using stainless steel. They were designed to hold large targets (see Fig. 3) such that they could be irradiated at high proton energies and obtain high production yields as a result. The station had a rail transport system installed, such that targets could be loaded and unloaded from a hot cell adjacent to the station.

In the late 1990s the focus turned away from commercial radionuclide production to the development of novel radionuclides for PET. This required some modifications to the target holder design such that it could hold degraders to decrease the beam energy as needed. Since many production routes for PET radiometals involve the use of enriched (and expensive) target material, the target capsule also needed to be redesigned (Fig. 5).

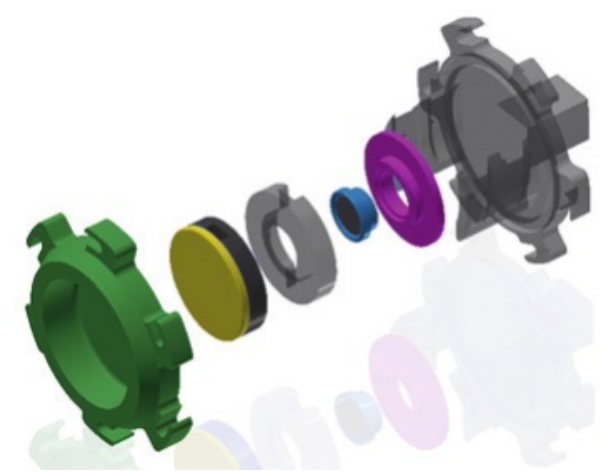

Fig. 5. Exploded view of target holder containing the target capsule and degrader (blue and yellow, respectively).

Because the proton beam at the IP2 irradiation station is only available for approximately 9 months of the year these days, this facility cannot be considered for use in any commercial setting. The system can, however, still be put to good use towards the development of novel, non-standard radiometals. Previously, the station was used to produce the likes of ${ }^{18} \mathrm{~F}^{[15]}$ and ${ }^{124} \mathrm{I}^{[16]}$ in a novel way by utilizing its higher beam energy, as well as ${ }^{67} \mathrm{Cu},{ }^{[17-19]}$ ${ }^{82} \mathrm{Sr},{ }^{52} \mathrm{Fe},{ }^{[20]}{ }^{76} \mathrm{Br}{ }^{[21]}$ and ${ }^{68} \mathrm{Ge}$, but these activities were halted well over a decade ago. With the increased popularity of PET for the diagnosis of cancer, as a result of its superior image resolution over Single Photon Emission Computed Tomography (SPECT), the strategy of the station's use was adjusted to meet the growing demand for new positron-emitting radionuclides. To meet the re- 
vised strategy of IP2's use, it was necessary to degrade the proton beam from $72 \mathrm{MeV}$ to the desired energy of the radiometal to be produced using $\mathrm{Nb}$ discs (Fig. 6). Currently, IP2 gleans $\sim 50 \mu \mathrm{A}$ protons from Injector II, by means of a beam splitter placed along the beam line between Injector II and the Ring cyclotron (Fig. 4). Once developed, these proofs-of-principle can then be put into practice at partner facilities.

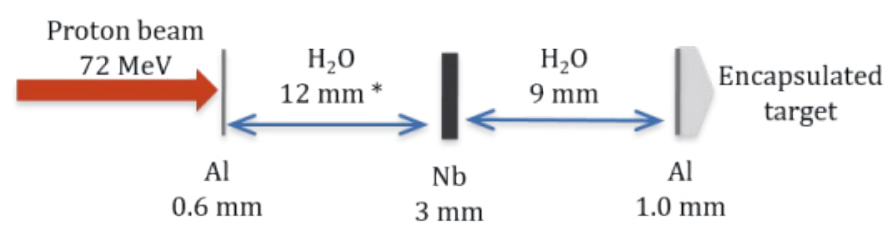

Fig. 6. A representative diagram of the degrading of the $72 \mathrm{MeV}$ proton beam at PSI's IP2 irradiation station. The water jacket between the encapsulated target and the degrader remains constant.

In today's world of nuclear medicine, the concept of using radiometals for PET has broadened enormously and many innovative ideas with regard to radiometals use, along with development of biomolecules for the nuclides to label to, have occurred over the last decade. The Radionuclide Development group at Paul Scherrer Institute currently contributes to this form of research by developing the radiometals below.

\subsection{1 ${ }^{44} \mathrm{Sc}$}

${ }^{44} \mathrm{Sc}$ is seen as a potentially ideal radiometal for PET, as its half-life $\left(\mathrm{T}_{1 / 2}=3.97 \mathrm{~h}\right)$ is longer and resolution is better than ${ }^{68} \mathrm{Ga}$ (currently the most popular radiometal in use for PET; $\mathrm{T}_{1 / 2}$ $=68 \mathrm{~min}$ ). Initially, ${ }^{44} \mathrm{Sc}$ was obtained from a ${ }^{44} \mathrm{Ti} /{ }^{44} \mathrm{Sc}$ generator, where the parent $\left({ }^{44} \mathrm{Ti}\right)$ radionuclide would decay into the daughter $\left({ }^{44} \mathrm{Sc}\right)$ and the daughter would be eluted (or 'milked') from the generator. ${ }^{[22]}$ The disadvantage of such a system is two-fold. Firstly, the production rate for ${ }^{44} \mathrm{Ti}$ (from $\mathrm{Sc}$ ) is extremely low, thereby only delivering low-activity generators from which ${ }^{44} \mathrm{Sc}$ can be obtained. Secondly, the half-life of the parent nuclide, ${ }^{44} \mathrm{Ti}$ $\left(T_{1 / 2}=60 \mathrm{a}\right)$, is such that breakthrough from the generator could have disastrous consequences in a clinical setting. ${ }^{[23]}$

Alternatively, ${ }^{44} \mathrm{Sc}$ can be produced at a cyclotron via the ${ }^{44} \mathrm{Ca}(\mathrm{p}, \mathrm{n}){ }^{44} \mathrm{Sc}$ nuclear reaction. Cross section measurements determine the ideal energy for irradiation to produce the maximum yield of product, as well as minimizing potential side products. An example can be seen in (Fig. 7). Initially, enriched $\mathrm{CaCO}_{3}$ targets were pressed onto graphite and irradiated with $\sim 11 \mathrm{MeV}^{3}$ protons at $50 \mu \mathrm{A}$ beam intensity, however, these targets were not homogeneous and $6 \mathrm{~mm}$ pressed pure carbonate targets were developed to replace them. It was subsequently discovered that the carbonate targets would easily dissociate in the beam, with the release of radioactive gas, indicating the necessity to further develop the targetry. Enriched carbonate targets were converted to oxide targets, which were found to be far more robust under irradiation conditions. [24]

Irradiated targets were dissolved in hydrochloric acid and loaded onto a column containing DGA extraction resin, where the ${ }^{44} \mathrm{Sc}$ was retained and the $\mathrm{Ca}$ target material passed through the system. This was collected separately and subsequently recycled to make new targets. The desired ${ }^{44} \mathrm{Sc}$ was eluted with dilute hydrochloric acid and concentrated onto a second, smaller, resin column. The final product was eluted from this second column (Fig. 8) in a small volume such that it could be used effectively for preclinical ${ }^{[25]}$ and clinical studies. ${ }^{[26]}$

\subsection{2 ${ }^{43} \mathrm{Sc}$}

While ${ }^{44} \mathrm{Sc}$ is an attractive PET radionuclide, it has the disadvantage of the emission of a $\gamma$-ray at $1157 \mathrm{keV}$, with almost $100 \%$

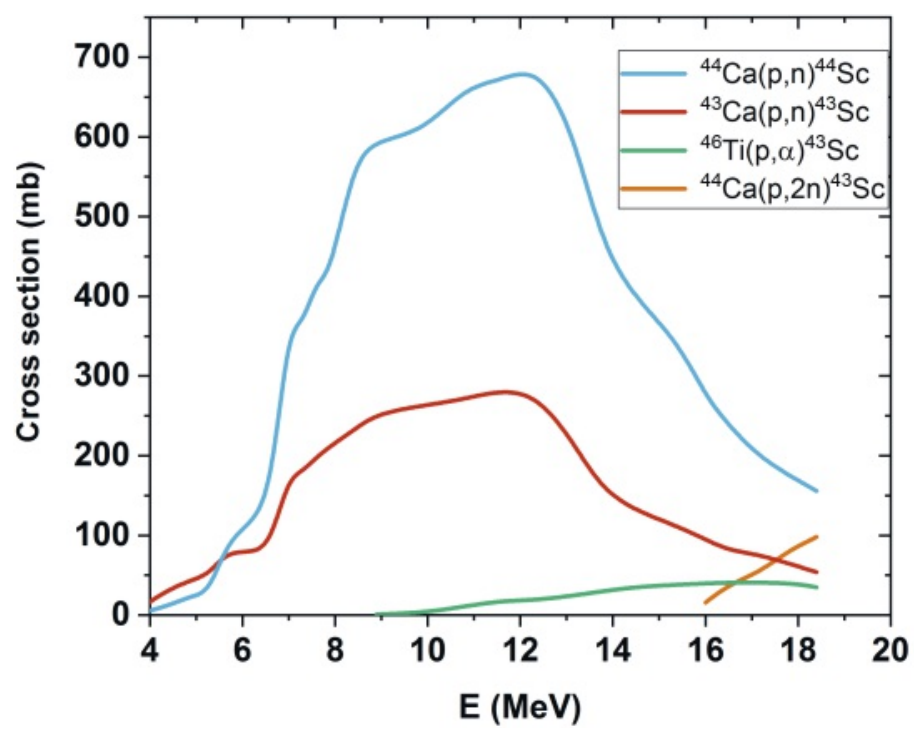

Fig. 7. Proton cross sections for different nuclear reactions to produce ${ }^{44} \mathrm{Sc}$ and ${ }^{43} \mathrm{Sc}$, respectively. It can be seen that the cross section to produce ${ }^{44} \mathrm{Sc}$ is considerably higher than that for ${ }^{43} \mathrm{Sc}$ when using proton irradiation. Adapted from ref. [29].

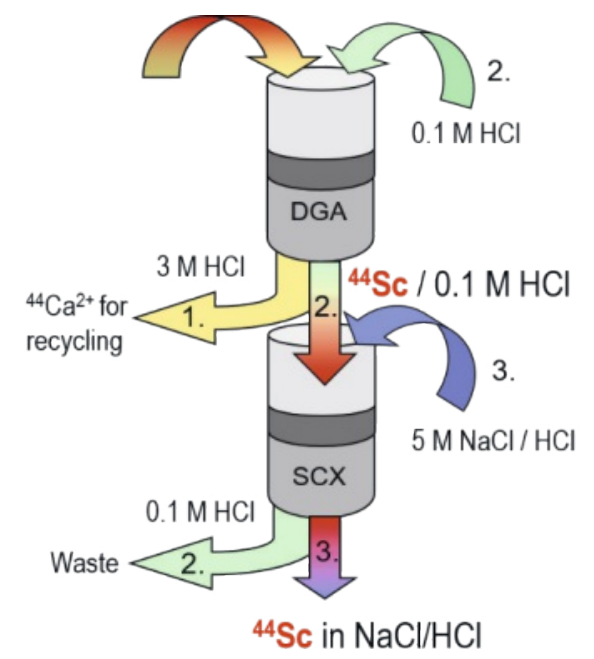

Fig. 8. Schematic diagram of the ${ }^{44} \mathrm{Sc}$ chemical separation process.

intensity, having implications on radiation protection and image quality. A suggested alternative is ${ }^{43} \mathrm{Sc}$, with a similar half-life $\left(\mathrm{T}_{1 / 2}=3.89 \mathrm{~h}\right)$, but its $\gamma$-emission at $373 \mathrm{keV}$ is at $22.5 \%$ intensity, thereby, decreasing potential dose to the clinical operator and patient and slightly improving image resolution. ${ }^{[27,28]}$

${ }^{43} \mathrm{Sc}$ is more difficult to produce than its ${ }^{44} \mathrm{Sc}$ counterpart. The production cross sections, using the two most popular production routes with proton irradiation, are lower than for ${ }^{44} \mathrm{Sc}^{[29]}$ (Fig. 7) and the means to produce it is more expensive, thereby, making it less attractive to introduce into the clinic. It is produced at IP2 via the ${ }^{46} \mathrm{Ti}(\mathrm{p}, \alpha)^{43} \mathrm{Sc}$ nuclear reaction (degrading to $\sim 13.4 \mathrm{MeV}$ protons) or via the ${ }^{43} \mathrm{Ca}(\mathrm{p}, \mathrm{n}){ }^{43} \mathrm{Sc}$ nuclear reaction using similar irradiation conditions to that of ${ }^{44} \mathrm{Sc}$ production. [27]

The ${ }^{46} \mathrm{Ti}_{2} \mathrm{O}_{3}$ was initially reduced to $\mathrm{Ti}$ powder and then pressed into a graphite pellet for irradiation. The beam intensity was reduced to $20 \mu \mathrm{A}$ for optimum irradiation and target dissolution conditions. The Ti target was dissolved in concentrated hydrochloric acid, diluted slightly and passed through a column containing DGA extraction resin, where the Sc was retained and the Ti collected for recycling. The Sc was eluted in dilute hydrochloric acid and concentrated on a small SCX cation exchange column. The product was eluted in a small volume of hydrochlo$\mathrm{ric}$ acid/sodium chloride solution. While radionuclidic purity was 
high (>98\%), the separation was time-consuming and the yields poor, due to the low cross section of the ${ }^{46} \mathrm{Ti}(\mathrm{p}, \alpha)^{43} \mathrm{Sc}$ nuclear reaction. ${ }^{[27]}$

${ }^{43} \mathrm{Sc}$ production and separation from enriched $\mathrm{Ca}$ were performed as for ${ }^{44} \mathrm{Sc}$. Enrichment of ${ }^{43} \mathrm{Ca}$ determines radionuclidic purity of the product, in this case, $57 \%$ enriched ${ }^{43} \mathrm{Ca}$ produced $66.6 \%{ }^{43} \mathrm{Sc}$ and $33.3 \%{ }^{44} \mathrm{Sc}$. The chemical separation, in comparison to the Ti route, was simple and fast and the yield considerably higher, however, the target material is prohibitively expensive.

A more recent approach was taken to make this radionuclide, namely, via the ${ }^{44} \mathrm{Ca}(\mathrm{p}, 2 \mathrm{n}){ }^{43} \mathrm{Sc}$ production route. ${ }^{\left[{ }^{30]}\right.}$ Enriched ${ }^{44} \mathrm{CaO}$ pellets were encapsulated and irradiated as for ${ }^{44} \mathrm{Sc}$, instead, the $\mathrm{Nb}$ degrader used was thinner - coinciding with $\sim 26.4 \mathrm{MeV}$ protons. High yields were obtained, with $>70 \%{ }^{43} \mathrm{Sc}$ obtained along with $30 \%$ co-produced ${ }^{44} \mathrm{Sc}$. The product was of high chemical purity, confirmed by the ability to use it for high-specific radiolabelling (up to $25 \mathrm{MBq} / \mathrm{nmol}$ molar activity) of biomolecules.[2]

\subsection{3 ${ }^{64} \mathrm{Cu}$}

${ }^{64} \mathrm{Cu}$ is a medically-interesting PET radionuclide with a longer half-life than many of its radiometal counterparts used for similar purposes $\left(\mathrm{T}_{1 / 2}=12.7 \mathrm{~h}\right)$ and is known to produce images of high resolution. It is produced via the ${ }^{64} \mathrm{Ni}(\mathrm{p}, \mathrm{n})^{64} \mathrm{Cu}$ nuclear reaction and the target material required to produce this radionuclide is expensive. A thin layer of enriched $\mathrm{Ni}$ is electroplated onto 0.5 $\mathrm{mm}$ thick gold foils as means of target preparation for irradiation (Fig. 9B).

The irradiated ${ }^{64} \mathrm{Ni}$ (irradiated with a degraded proton beam similar to that for ${ }^{44} \mathrm{Sc}$ ) was dissolved from the gold foil in hydrochloric acid and subsequently diluted to obtain a concentration of $0.1 \mathrm{M}$ hydrochloric acid and $60 \%$ acetone mixture. This resultant solution was passed through a column containing AG MP-50 macroporous cation exchange resin, where both $\mathrm{Cu}$ and $\mathrm{Ni}$ were retained. The concentration of acetone was adjusted to elute the ${ }^{64} \mathrm{Cu}$ final product first, the acetone concentration further adjusted to elute the Co radio-impurities separately, before the enriched $\mathrm{Ni}$ is eluted in hydrochloric acid and collected for recycling. ${ }^{[31]}$ The radionuclide has been used extensively for preclinical studies. ${ }^{[32,33]}$

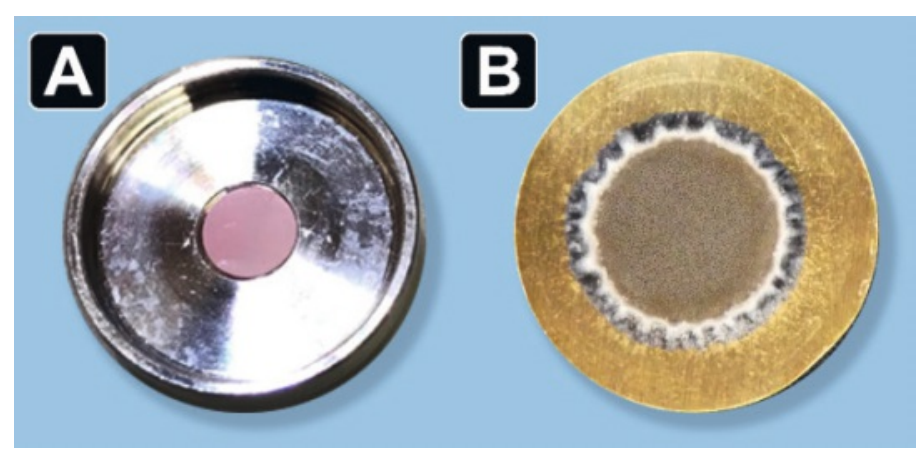

Fig. 9. (A) Pressed oxide target, $6 \mathrm{~mm}$ in diameter, nestled in the indentation of an $\mathrm{Al}$ (99.5\% pure) capsule. (B) Ni metal electroplated on gold $\operatorname{disc}(\varnothing 19 \mathrm{~mm})$.

\subsection{4 ${ }^{165} \mathrm{Er}$}

${ }^{165} \mathrm{Er}$ is a pure Auger-emitting radiolanthanide and can be regarded as an interesting option towards targeted radionuclide therapy. It emits no $\gamma$-rays but can, nevertheless, be detected by its low energy X-rays. It can be produced by means of low-energy protons via the ${ }^{165} \mathrm{Ho}(\mathrm{p}, \mathrm{n}){ }^{165} \mathrm{Er}$ nuclear reaction (referred to as the 'direct' route) or at higher proton energies via the ${ }^{166} \mathrm{Er}(\mathrm{p}, 2 \mathrm{n}){ }^{165} \mathrm{Tm} \rightarrow{ }^{165} \mathrm{Er}$ nuclear reaction (referred to as the 'indirect' production route). ${ }^{[34]}$

The direct route, based on naturally mononuclidic Ho (thus, comparatively inexpensive), utilized pressed $\mathrm{Ho}_{2} \mathrm{O}_{3}$ targets 13 $\mathrm{mm}$ in diameter, as the $6 \mathrm{~mm}$ diameter target produced relatively low activities of product. The beam settings had to be adjusted accordingly, however, the beam intensity had to be reduced to $20 \mu \mathrm{A}$, as any higher intensity had decreased the production yield. The same irradiation parameters were applied to the indirect production route, however, a 6-mm target was prepared for the irradiation of enriched target material ( ${ }^{166} \mathrm{Er} 98.1 \%$ enrichment), thereby, requiring a more focused proton beam than for the Ho irradiation (Fig. 9A). ${ }^{[35]}$

A chemical separation method based on that determined for ${ }^{161} \mathrm{~Tb}$ production at PSI (see below) ${ }^{[36]}$ was adopted for ${ }^{165} \mathrm{Er}$.

\subsection{The Swiss Neutron Source (SINQ)}

The thought of having a Swiss spallation neutron source onsite was hatched soon after the successful commissioning of the $590 \mathrm{MeV}$ proton cyclotron at SIN in the 1970s. ${ }^{[37]}$ The spallation process typically provides a thermal neutron flux of the order of $10^{14} \mathrm{n} \mathrm{cm}^{-2} \mathrm{~s}^{-1} \mathrm{~mA}^{-1}$. The concept was presented in 1978 and was met with approval from a meeting consisting of many international constituents. It was agreed that a continuous neutron source would be very important for condensed matter research. After a considerable time spent on designing (and redesigning) the facility and obtaining the required funding, the construction of SINQ began in 1988 and the first neutron beam was produced in early December 1996. It uses the remaining beam $(\sim 70 \%)$ from the $590 \mathrm{MeV}$ Ring cyclotron. This beam is deflected into an extended beam line towards a final target designed to stop the proton beam - a Pb 'beam dump' which became the target for SINQ. ${ }^{[7]}$

SINQ houses a 'rabbit' system for neutron irradiation of materials inserted into a beam tube. ${ }^{[38]}$ Two pairs of rabbit system tubes reach into the volume of the moderator vessel. The one pair of tubes closest to the target (PNA) is utilized for radionuclide production and development, which includes the production of radioactive tracers towards developing chemical separation methods (thermal neutron flux: $2 \times 10^{13} \mathrm{n} \mathrm{cm}^{-2} \mathrm{~s}^{-1} \mathrm{~mA}^{-1}$ ). Nuclides produced there in the past include ${ }^{24} \mathrm{Na},{ }^{82} \mathrm{Br},{ }^{42} \mathrm{~K},{ }^{175 / 181} \mathrm{Hf},{ }^{131} \mathrm{I},{ }^{60} \mathrm{Co},{ }^{198} \mathrm{Au}$, which were used for trace experiments. Over the last decade, however, PNA has been predominantly used for the development and upscale of ${ }^{161} \mathrm{~Tb}$ production. ${ }^{[39]}$ Target material, sealed in a quartz ampoule, is placed into in an $\mathrm{Al}$ capsule, which is welded shut before irradiation (Fig. 10).
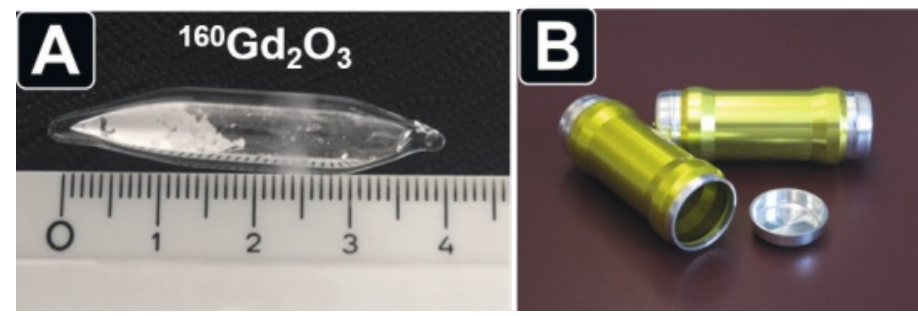

Fig. 10. (A) Quartz ampoule containing enriched ${ }^{160} \mathrm{Gd} \mathrm{O}_{3}$ for ${ }^{161} \mathrm{~Tb}$ production. The sealed ampoule is placed in an Al capsule (B), which is welded shut in preparation for neutron irradiation at SINQ.

\section{2. ${ }^{161} \mathrm{~Tb}$}

Terbium-161 decays with a half-life of $6.95 \mathrm{~d}$ (recently precisely determined ${ }^{[40]}$ ) by the emission of medium-energy $\beta^{-}$particles $\left(\mathrm{E}_{\beta}\right.$ average $\left.=154 \mathrm{keV}\right)$ and $\gamma$-radiation $\left(\mathrm{E}_{\gamma}=48 \mathrm{keV}(\mathrm{I}=\right.$ $17.0 \%), 75 \mathrm{keV}(\mathrm{I}=10.2 \%))$. While ${ }^{177} \mathrm{Lu}$ is currently regarded as the benchmark in clinical radionuclide therapy, it is believed that ${ }^{161} \mathrm{~Tb}$ would be more effective as a result of its greater number of Auger and conversion electrons, which could address the issue of small metastases and single cancer cells. ${ }^{[28,41,42]}$

It is produced via the ${ }^{160} \mathrm{Gd}(\mathrm{n}, \gamma)^{161} \mathrm{Gd} \rightarrow{ }^{161} \mathrm{~Tb}$ nuclear reaction from enriched ${ }^{160} \mathrm{Gd}$ target material. ${ }^{[36]}$ Initially, the target am- 
poule/capsule (Fig. 10B) was irradiated at SINQ for a period of three weeks, before removal and processing.

With $\mathrm{Tb}$ and Gd being two neighboring lanthanides, their efficient separation requires well optimized conditions. The target material is dissolved in acid and ${ }^{161} \mathrm{~Tb}$ separated from enriched ${ }^{160} \mathrm{Gd}$ by passing it through cation exchange resin, ${ }^{[36]}$ before the product is eluted and concentrated using LN3 extraction resin. The desired ${ }^{161} \mathrm{~Tb}$ is finally eluted in a small volume of dilute $\mathrm{HCl}$ in a quality that ensures labelling of biomolecules, with a DOTA chelator, at molar activities $>100 \mathrm{MBq} / \mathrm{nmol}$. This radionuclide has been used extensively in preclinical studies. ${ }^{[28,41]}$ The current limit of $20 \mathrm{GBq}{ }^{161} \mathrm{~Tb}$ that can be shipped due to the restriction of the international air transport agency (IATA) currently prevents the translation of ${ }^{161} \mathrm{~Tb}$ to clinical stage (a patient dose is the equivalent of $\sim 7 \mathrm{GBq}$ post processing and labelling). The irradiation facility (see section 4.1) is a long distance away from the processing laboratory and the irradiated raw material requires transportation for it to be chemically processed. Once this limit is lifted (expected in 2021), it will be feasible to make ${ }^{161} \mathrm{~Tb}$ available in activities sufficient for clinical application.

\section{PSI's Radionuclide Development Group and its Partner Network}

\subsection{Nuclear Research Reactors}

While development work with regard to neutron irradiation is performed at SINQ, partner nuclear reactor facilities such as Institut Laue-Langevin (ILL, Grenoble, France) and the South African Nuclear Energy Corporation (Necsa, Pelindaba, South Africa) play a vital role in the upscale of these productions, as their neutron flux is considerably higher than PSI's SINQ facility. This has been successfully performed with ${ }^{161} \mathrm{~Tb}$, in particular.[36]

\subsection{1 ${ }^{47} \mathrm{Sc}$}

Based on development of ${ }^{44} \mathrm{Sc}$, the concept of developing ${ }^{47} \mathrm{Sc}$ as its matched pair therapeutic nuclide was investigated. ${ }^{[43]}$ Initially, the use of enriched $\mathrm{CaCO}_{3}$ target material was used, sealed in a quartz ampoule and sent to ILL for high neutron flux irradiation of the target. The ${ }^{47} \mathrm{Sc}$ was produced via the ${ }^{46}(\mathrm{n}, \gamma){ }^{47} \mathrm{Ca} \rightarrow{ }^{47} \mathrm{Sc}$ nuclear reaction. The ampoule was subsequently shipped back to PSI for processing, following a similar chemical separation process as that practiced for ${ }^{44} \mathrm{Sc}$. The ${ }^{47} \mathrm{Ca}$ was separated from the desired product and left to decay to ${ }^{47} \mathrm{Sc}$, such that the chemical separation process could be repeated every third day and the product used for preclinical application. ${ }^{[44]}$ The target material was subsequently changed to that of enriched ${ }^{46} \mathrm{CaO}$ (less radiation-induced degassing than carbonate) and the chemical separation process slightly adjusted. ${ }^{[45]}$

\subsection{Isotope Separation OnLine (ISOL)}

\section{2..$^{149 / 152 / 155 T b}$}

A campaign at ISOLDE, CERN (Switzerland), spanning nine years resulted in collections of ${ }^{149} \mathrm{~Tb},{ }^{[46,47]}{ }^{152} \mathrm{~Tb}^{[48-50]}$ and ${ }^{155} \mathrm{~Tb}^{[51]}$ radioisotopes, ${ }^{[52]}$ respectively, for use towards preclinical application. Overnight collections of the respective mass number were performed by proton-induced spallation in a tantalum target, followed by ionization of the spallation products and mass separation. The respective mass isobars were implanted into zinc-coated gold foils and the collected sample left to stand, to allow shortlived radionuclides to decay before transport to PSI. The Zn was dissolved from the Au foil using dilute ammonium nitrate, and the isobars loaded onto a macroporous cation exchange resin. The Tb radioisotope was separated from impurities using gradient elution with $\alpha$-hydroxy-isobutyric acid ( $\alpha$-HIBA, $\mathrm{pH} 4.7)$ and used directly for radiolabelling and subsequent preclinical work. ${ }^{152} \mathrm{~Tb}$ was used in a clinical setting in Germany. ${ }^{[48,53,54]}$

\subsection{Medical Cyclotron Facilities}

The PET radiometals described above are developed at PSI's IP2 irradiation station as a proof of concept. Once the development reaches an advanced stage, it is necessary to be able to convert the concept such that it can be used at a medical cyclotron. PSI's Radionuclide Development group has close collaboration with the Laboratory of High Energy Physics (Albert Einstein Center for Fundamental Physics) at the University of Bern (UniBe), as well as the Center of Radiopharmaceutical Sciences at ETHZ for this purpose.

Targets for irradiation to produce PET radiometals were developed using PSI's IP2 irradiation station, however, they can easily be utilized at the irradiation facilities at UniBe and ETHZ. Enriched oxide targets have been designed to be $6 \mathrm{~mm}$ in diameter and $0.5 \mathrm{~mm}$ thick (Fig. 9A), while for ${ }^{64} \mathrm{Cu}$ production an $\mathrm{Au}$ foil, $19 \mathrm{~mm}$ in diameter, is plated with enriched Ni (Fig. 9B) ${ }^{[31]}$. This concept has been slightly adapted for use at the cyclotron facility at UniBe, however, the capsule and target design (Fig. 9A) has been used directly towards the design of the solid target station (with an IBA Cyclone 18/9) recently implemented at ETHZ.

The ${ }^{44} \mathrm{Sc}$ production system was implemented at the medical cyclotron facility at UniBe, which houses an IBA Cyclone 18/18 with a solid target station. The enriched $\mathrm{CaO}$ pellet was the same, however, the encapsulation design was customized to fit into the target station available there. ${ }^{[24]}$

\section{Conclusions and Outlook}

Radionuclide production and development in Switzerland, and PSI in particular, is rich in history and dates back to the very beginning of nuclear energy in Switzerland. PSI, in its current and previous guises, has contributed substantially to the world of radiochemistry, radiopharmacy and nuclear medicine since the 1950s. Swiss scientists have watched the facilities in use metamorphose over the decades; there are plans to further evolve using cutting edge technology, thereby, keeping the Institute at the forefront of the field. ${ }^{[2,55]}$

Currently, the IP2 target station at PSI is an effective tool towards proof-of-concept development of exotic radionuclides in Switzerland. Its effectiveness has resulted in enabling PET radiometals production by irradiation at medical cyclotrons, with the target capsule concept being retained for the solid target station facility at ETHZ. SINQ has also proven to be an effective tool for the development of ${ }^{161} \mathrm{~Tb}$, with the irradiation being up-scaled at partner neutron irradiation facilities. Further updates to target irradiation, as well as ideas towards facility upgrades, are being tabled which, it is anticipated, will secure radionuclide development and radiochemistry in Switzerland for the foreseeable future.

\section{Acknowledgements}

The authors acknowledge the contributions of PSI's Radionuclide Production and Maintenance group, over the years, and Walter Hirzel, Alexander Sommerhalder, Muhamet Djelili and André Isenschmid, in particular. PSI's Radionuclide Development group is grateful for collaboration with PSI's Nuclide Chemistry group, and PD Dr. Cristina Müller in particular, towards bringing the radionuclides developed into a preclinical setting.

Received: August 7, 2020

[1] Z. Talip, C. Favaretto, S. Geistlich, N. P. van der Meulen, Molecules 2020 $25,966$.

[2] N. P. van der Meulen, R. Eichler, P. V. Grundler, R. Hasler, W. Hirzel, S. Joray, D. C. Kiselev, R. Sobbia, A. Sommerhalder, Z. Talip, H. Zhang, in JACoW Publishing, 2019, https://doi.org/10.18429/JACoW-Cyclotrons2019-TUA03.

[3] Reaktor AG, 'Geschaeftsbericht 1959', Wuerenlingen, Aargau.

[4] T. Wildi, University of Zurich, Zurich, 2003.

[5] F. Alder, A. Colomb, A. Fritzsche, P. Schmid, F. Staub, W. Zuenti, 'Description of the Swiss Research Reactors', Wuerenlingen, 1957. 
[6] R. H. Weinreich, I. Jegge, J. Willax, H. Reist, H. W. Oehninger, in 'Radiopharmaceuticals and labelled compounds 1984', IAEA, Tokyo, 1985 p. 55.

[7] 'User's guide: Accelerator facilities', Villigen and Wuerenlingen, 1994

[8] R. Maag, R. Weinreich, in 'Proceedings of IVth International Workshop on Targetry and Target Chemistry', Paul Scherrer Institut, Villigen, Switzerland, 1991, p. 68.

[9] Swiss Federal Institute for Reactor Research, 'EIR Jahresbericht 1974', Wuerenlingen, 1975

[10] Swiss Federal Institute for Reactor Research, 'EIR Jahresbericht 1975', 1976.

[11] R. Pellikka, I. Huszar, J. Jegge, R. Schwarzbach, P. Blauenstein, P. A. Schubiger, in 'VII. Boettstein Colloqium; PET-Radiopharmaceuticals at PSI: Achievement and future prospects', Paul Scherrer Institute, Boettstein, 1993, p.13.

[12] 'SIN Jahresbericht 1987', Villigen, 1987.

[13] R. Schwarzbach, G. Reddy, J. Jegge, I. Huszar, in 'VII. Boettstein Colloqium; PET-Radiopharmaceuticals at PSI: Achievement and future prospects', PSI, Boettstein, Switzerland, 1993, p. 10.

[14] M. Seidel, S. Adam, A. Adelmann, C. Baumgarten, R. Doelling, in Conf. Proc. C 100523 (2020) TUYRA03, 2010

[15] R. Schwarzbach, I. Huszar, J. Jegge, P. A. Schubiger, in, 'Proceedings of Fifth International Workshop on Targetry and Target Chemistry (WTTC)', Upton, New York, 1993, p. 77.

[16] K. E. J. Weinreich, in 'Proceedings of Sixth International Workshop on Targetry and Target Chemistry', Vancouver, 1995, p. 84

[17] R. Schwarzbach, K. Zimmermann, P. Bläuenstein, A Smith, P. A. Schubiger, Appl. Radiat. Isot. 1995, 46, 329 https://doi.org/10.1016/0969-8043(95)00010-B.

[18] R. Schwarzbach, K. Zimmermann, I. Novak-Hofer, P. A Schubiger, J. Labell. Comp. Radiopharm. 2001, 44, S809, https://doi.org/10.1002/jlcr.25804401284.

[19] I. Novak-Hofer, P. A. Schubiger, Eur. J. Nucl. Med. Mol. Imag. 2002, 29, 821, https://doi.org/10.1007/s00259-001-0724-y.

[20] P. Smith-Jones, R. Schwarzbach, R. Weinreich, Radiochim. Acta 1990, 50, 33 , https://doi.org/10.1524/ract.1990.50.12.33.

[21] J. E. Ryser, P. Bläuenstein, N. Rémy, R. Weinreich, P. H. Hasler I. Novak-Hofer, P. A. Schubiger, Nucl. Med. Biol. 1999, 26, 673, https://doi.org/10.1016/S0969-8051(99)00042-6.

[22] F. Roesch, Curr. Radiopharm. 2012, 5, 187.

[23] N. P. van der Meulen, M. Bunka, K. A. Domnanich, C. Müller, S. Haller, C. Vermeulen, A. Türler, R. Schibli, Nucl. Med. Biol. 2015, 42, 745, https://doi.org/10.1016/j.nucmedbio.2015.05.005.

[24] N. P. van der Meulen, R. Hasler, Z. Talip, P. V. Grundler, C. Favaretto, C Umbricht, C. Mueller, G. Dellepiane, T. S. Carzaniga, S. Braccini, Molecules 2020, 25, 4706 .

[25] K. A. Domnanich, C. Müller, R. Farkas, R. M. Schmid, B. Ponsard, R. Schibli, A. Türler, N. Van der Meulen, EJNMMI Radiopharm. Chem. 2016 , 8, 19, https://doi.org/10.1186/s41181-016-0013-5.

[26] A. Singh, N. P. van der Meulen, C. Muller, I. Klette, H. R. Kulkarni, A. Turler, R. Schibli, R. P. Baum, Cancer Biother. Radiopharm. 2017, 32, 124 , https://doi.org/10.1089/cbr.2016.2173.

[27] K. A. Domnanich, R. Eichler, C. Müller, S. Jordi, V. Yakusheva, S. Braccini, M. Behe, R. Schibli, A. Türler, N. P. van der Meulen, EJNMMI Radiopharm. Chem. 2017, 2, 14, https://doi.org/10.1186/s41181-017-0033-9.

[28] C. Müller, K. A. Domnanich, C. A. Umbricht, N. P. van der Meulen, Brit. J. Radiology 2018, 91, 20180074, https://doi.org/10.1259/bjr.20180074.

[29] T. S. Carzaniga, M. Auger, S. Braccini, M. Bunka, A. Ereditato, K. P. Nesteruk, P. Scampoli, A. Türler, N. Van der Meulen, Appl. Radiat. Isot. 2017, 129, 96.

[30] N. Van der Meulen, R. Hasler, Nucl. Med. Biol. 2019, 72-73, S9, https://doi.org/10.1016/S0969-8051(19)30215-X.

[31] N. P. van der Meulen, R. Hasler, A. Blanc, R. Farkas, M. Benešová, Z Talip, C. Müller, R. Schibli, J. Label. Comp. Radiopharm. 2019, 62, 460, https://doi.org/10.1002/jlcr.3730.

[32] R. Farkas, K. Siwowska, S. M. Ametamey, R. Schibli, N. P. van der Meulen, C. Müller, Mol. Pharma. 2016, 13, 1979, https://doi.org/10.1021/acs.molpharmaceut.6b00143.

[33] C. A. Umbricht, M. Benešová, R. Hasler, R. Schibli, N. P. van der Meulen, C. Müller, Mol. Pharma. 2018, 15, 5556, https://doi.org/10.1021/acs.molpharmaceut.8b00712.

[34] N. Gracheva, T. S. Carzaniga, R. Schibli, S. Braccini, N. P. van der Meulen, Appl. Radiat. Isot. 2020, 159, 109079 , https://doi.org/10.1016/j.apradiso.2020.109079.

[35] N. Gracheva, R. Schibli, N. P. van der Meulen, 'Production and purification of the pure Auger electron emitter Erbium-165', Villigen-PSI, 2019.
[36] N. Gracheva, C. Müller, Z. Talip, S. Heinitz, U. Köster, J. R. Zeevaart, A. Vögele, R. Schibli, N. P. van der Meulen, EJNMMI Radiopharm. Chem. 2019, 4, 12, https://doi.org/10.1186/s41181-019-0063-6.

[37] A. Furrer, in 'Swiss Neutron News', Swiss Neutron Scattering Society, 2017, No. 49, p. 6

[38] W. Wagner, Y. Dai, H. Glasbrenner, H.-U. Aebersold, J. Nucl. Mater. 2007, 361, 274, https://doi.org/10.1016/j.jnucmat.2006.12.010

[39] S. Lehenberger, C. Barkhausen, S. Cohrs, E. Fischer, J. Grünberg, A. Hohn, U. Köster, R. Schibli, A. Türler, K. Zhernosekov, Nucl. Med. Biol. 2011, 38 , 917, https://doi.org/10.1016/j.nucmedbio.2011.02.007.

[40] M. T. Durán, F. Juget, Y. Nedjadi, F. Bochud, P. V. Grundler, N. Gracheva, C. Müller, Z. Talip, N. P. van der Meulen, C. Bailat, Appl. Radiat. Isot. 2020 159, 109085, https://doi.org/10.1016/j.apradiso.2020.109085.

[41] C. Müller, C. A. Umbricht, N. Gracheva, V. J. Tschan, G Pellegrini, P. Bernhardt, J. R. Zeevaart, U. Köster, R. Schibli, N. P. van der Meulen, Eur. J. Nucl. Med. Mol. Imag. 2019, 46, 1919 , https://doi.org/10.1007/s00259-019-04345-0.

[42] C. Champion, M. A. Quinto, C. Morgat, P. Zanotti-Fregonara, E. Hindié, Theranostics 2016, 6, 1611, https://doi.org/10.7150/thno.15132.

[43] C. Muller, M. Bunka, S. Haller, U. Koster, V. Groehn, P. Bernhardt, N. van der Meulen, A. Turler, R. Schibli, J. Nucl. Med. 2014, 55, 1658 , https://doi.org/10.2967/jnumed.114.141614.

44] K. A. Domnanich, C. Müller, M. Benešová, R. Dressler, S. Haller, U. Köster, B. Ponsard, R. Schibli, A. Türler, N. Van der Meulen, EJNMMI Radiopharm. Chem. 2017, 2, 5, https://doi.org/10.1186/s41181-017-0024-x.

[45] K. Siwowska, P. Guzik, K. A. Domnanich, J. M. Monné Rodríguez, P. Bernhardt, B. Ponsard, R. Hasler, F. Borgna, R. Schibli, U. Köster, N. P. van der Meulen, C. Müller, Pharmaceutics 2019, 11, 424.

[46] C. Müller, C. Vermeulen, U. Köster, K. Johnston, A. Türler, R. Schibli, N. P. van der Meulen, EJNMMI Radiopharm. Chem. 2016, 1, 5, https://doi.org/10.1186/s41181-016-0008-2.

[47] C. A. Umbricht, U. Köster, P. Bernhardt, N. Gracheva, K. Johnston, R. Schibli, N. P. van der Meulen, C. Müller, Sci. Rep. 2019, 9, 17800 https://doi.org/10.1038/s41598-019-54150-w.

[48] C. Müller, A. Singh, C. A. Umbricht, H. R. Kulkarni, K. Johnston, M. Benešová, S. Senftleben, D. Müller, C. Vermeulen, R. Schibli, U. Köster, N. P. van der Meulen, R. P. Baum, EJNMMI Res. 2019, 9, 68 , https://doi.org/10.1186/s13550-019-0538-1.

[49] F. Cicone, S. Gnesin, T. Denoël, T. Stora, N. P. van der Meulen, C. Müller, C. Vermeulen, M. Benešová, U. Köster, K. Johnston, E. Amato, L. Auditore, G. Coukos, M. Stabin, N. Schaefer, D. Viertl, J. O. Prior, EJNMMI Res. 2019, 9, 53, https://doi.org/10.1186/s13550-019-0524-7.

[50] C. Müller, C. Vermeulen, K. Johnston, U. Köster, R. Schmid, A. Türler, N. P. van der Meulen, EJNMMI Res. 2016, 6, 35 , https://doi.org/10.1186/s13550-016-0189-4.

[51] C. Muller, E. Fischer, M. Behe, U. Koster, H. Dorrer, J. Reber, S. Haller, S. Cohrs, A. Blanc, J. Grunberg, M. Bunka, K. Zhernosekov, N. van der Meulen, K. Johnston, A. Turler, R. Schibli, Nucl. Med. Biol. 2014, 41, Suppl, e58, https://doi.org/10.1016/j.nucmedbio.2013.11.002.

[52] C. Müller, K. Zhernosekov, U. Köster, K. Johnston, H. Dorrer, A. Hohn, $J$. Nucl. Med. 2012, 53, https://doi.org/10.2967/jnumed.112.107540.

[53] J. Zhang, A. Singh, H. R. Kulkarni, C. Schuchardt, D. Müller, H.-J. Wester, T. Maina, F. Rösch, N. P. van der Meulen, C. Müller, H. Mäcke, R. P. Baum, Sem. Nucl. Med. 2019, 49, 422 , https://doi.org/10.1053/j.semnuclmed.2019.06.002.

[54] R. P. Baum, A. Singh, M. Benesova, C. Vermeulen, S. Gnesin, U. Koster, K. Johnston, D. Muller, S. Senftleben, H. R. Kulkarni, A. Turler, R. Schibli, J. O. Prior, N. P. van der Meulen, C. Muller, Dalton Trans. 2017, 46, 14638 , https://doi.org/10.1039/C7DT01936J.

[55] H. Zhang, R. Eichler, J. Grillenberger, W. Hirzel, S. Joray, D. C. Kiselev, N. P. van der Meulen, J. M. Schippers, J. Snuverink, R. Sobbia, A. Sommerhalder, Z. Talip, in JACoW Publishing, 2019, https://doi.org/10.18429/JACoW-Cyclotrons2019-WEB04.

\section{License and Terms}

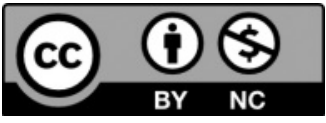

This is an Open Access article under the terms of the Creative Commons Attribution License CC BY_NC 4.0. The material may not be used for commercial purposes.

The license is subject to the CHIMIA terms and conditions: (http:// chimia.ch/component/sppagebuilder/?view = page \&id=12).

The definitive version of this article is the electronic one that can be found at https://doi.org/10.2533/chimia.2020.968 